\title{
Radio observations and spectral index study of the SNR G126.2+1.6
}

\author{
W. W. $\operatorname{Tian}^{1,2}$ and D. A. Leahy ${ }^{2}$ \\ 1 National Astronomical Observatories, CAS, Beijing 100012, PR China \\ e-mail: tww@bao.ac.cn \\ 2 Department of Physics \& Astronomy, University of Calgary, Calgary, Alberta T2N 1N4, Canada
}

Received 11 August 2005 / Accepted 11 October 2005

ABSTRACT

We present new images of the low radio surface brightness Supernova Remnant (SNR) G126.2+1.6, based on the $408 \mathrm{MHz}$ and $1420 \mathrm{MHz}$ continuum emission and the HI-line emission data of the Canadian Galactic Plane Survey (CGPS). We find the SNR's flux densities at $408 \mathrm{MHz}$ $(9.7 \pm 3.9 \mathrm{Jy})$ and $1420 \mathrm{MHz}(6.7 \pm 2.1 \mathrm{Jy})$ which have been corrected for flux densities from compact sources within the SNR. We list properties of the 19 brightest compact sources within G126.2+1.6 at both $408 \mathrm{MHz}$ and $1420 \mathrm{MHz}$. The integrated flux density based spectral index $\left(S_{v} \propto v^{-\alpha}\right)$ is $0.30 \pm 0.41$. The respective T-T plot spectral index is $0.30 \pm 0.08$. We also find spatial variations of spectral index within the SNR: 0.2-0.6. HI observations show structures probably associated with the SNR, i.e., features associated with the SNR's southeastern filaments in the radial velocity range of -33 to $-42 \mathrm{~km} \mathrm{~s}^{-1}$, and with its northwestern filaments in -47 to $-52 \mathrm{~km} \mathrm{~s}^{-1}$. This association suggests a distance of $5.6 \mathrm{kpc}$ for SNR G126.2+1.6. The estimated Sedov age for G126.2+1.6 is less than $2.1 \times 10^{5} \mathrm{yr}$.

Key words. ISM: individual objects: G126.2+1.6 - ISM: supernova remnants

\section{Introduction}

As the third of a continuing study of supernova remnants spectral index variation (Tian \& Leahy 2005; Leahy \& Tian 2005), the radio spectrum of the SNR G126.2+1.6 is studied in detail in the paper. This low radio surface brightness source was first detected and classified as a SNR by Reich et al. (1979). Some of its basic physical features are unclear, such as its distance and radio spectrum. Flux densities of G126.2+1.6 and respective spectral indices have been given previously (Reich et al. 2003; Joncas et al. 1989; Fürst et al. 1984) based on observations with a lower sensitivity than the current observations. Previous studies show that better flux density measurements are needed for G126.2+1.6. In the paper, we present the SNR's continuum images at higher sensitivity than previously at $408 \mathrm{MHz}$ and $1420 \mathrm{MHz}$ in order to determine its flux densities and spectral index. We investigate HI-line emission at various radial velocities for detecting interactions of the remnant with the surrounding gas and estimating its distance and age.

\section{Observations and analysis}

The continuum and HI emission data sets come from the CGPS, which is described in detail by Taylor et al. (2003). The data sets are mainly based on observations from the Synthesis Telescope (ST) of the Dominion Radio Astrophysical
Observatory (DRAO). The spatial resolution of the continuum images is better than $1^{\prime} \times 1^{\prime} \operatorname{cosec}(\delta)(\mathrm{HPBW})$ at $1420 \mathrm{MHz}$ and $3.4^{\prime} \times 3.4^{\prime} \operatorname{cosec}(\delta)$ at $408 \mathrm{MHz}$. The synthesized beam for the $\mathrm{HI}$ line images is as the same as for the continuum and the radial velocity resolution is $1.32 \mathrm{~km} \mathrm{~s}^{-1}$. DRAO ST observations are not sensitive to structures larger than an angular size scale of about $3.3^{\circ}$ at $408 \mathrm{MHz}$ and $56^{\prime}$ at $1420 \mathrm{MHz}$. Thus the CGPS includes data from the $408 \mathrm{MHz}$ all-sky survey of Haslam et al. (1982), sensitive to structure greater than $51^{\prime}$, and the Effelsberg $1.4 \mathrm{GHz}$ Galactic plane survey of Reich et al. $(1990,1997)$, sensitive to structure with resolution 9.4' for large scale emission (the single-dish data are freely available by http://www.mpifr-bonn.mpg.de/survey.html). The low-order spacing HI data is from the single-antenna survey of the CGPS area (Higgs \& Tapping 2000) with resolution of $36^{\prime}$. See Taylor et al. (2003) for detail of the method of combining the synthesis telescopes and single dish observations.

We analyze the continuum and HI images of G126.2+1.6 and determine its flux densities and distance using the DRAO export software package. For G126.2+1.6, integrated flux density's errors are found by comparing results for several different choices of background region. For compact sources, the flux densitiy's errors are taken as the formal Gaussian fit errors. The influence of compact sources within the SNR is reduced by employing similar methods to Tian \& Leahy (2005). 

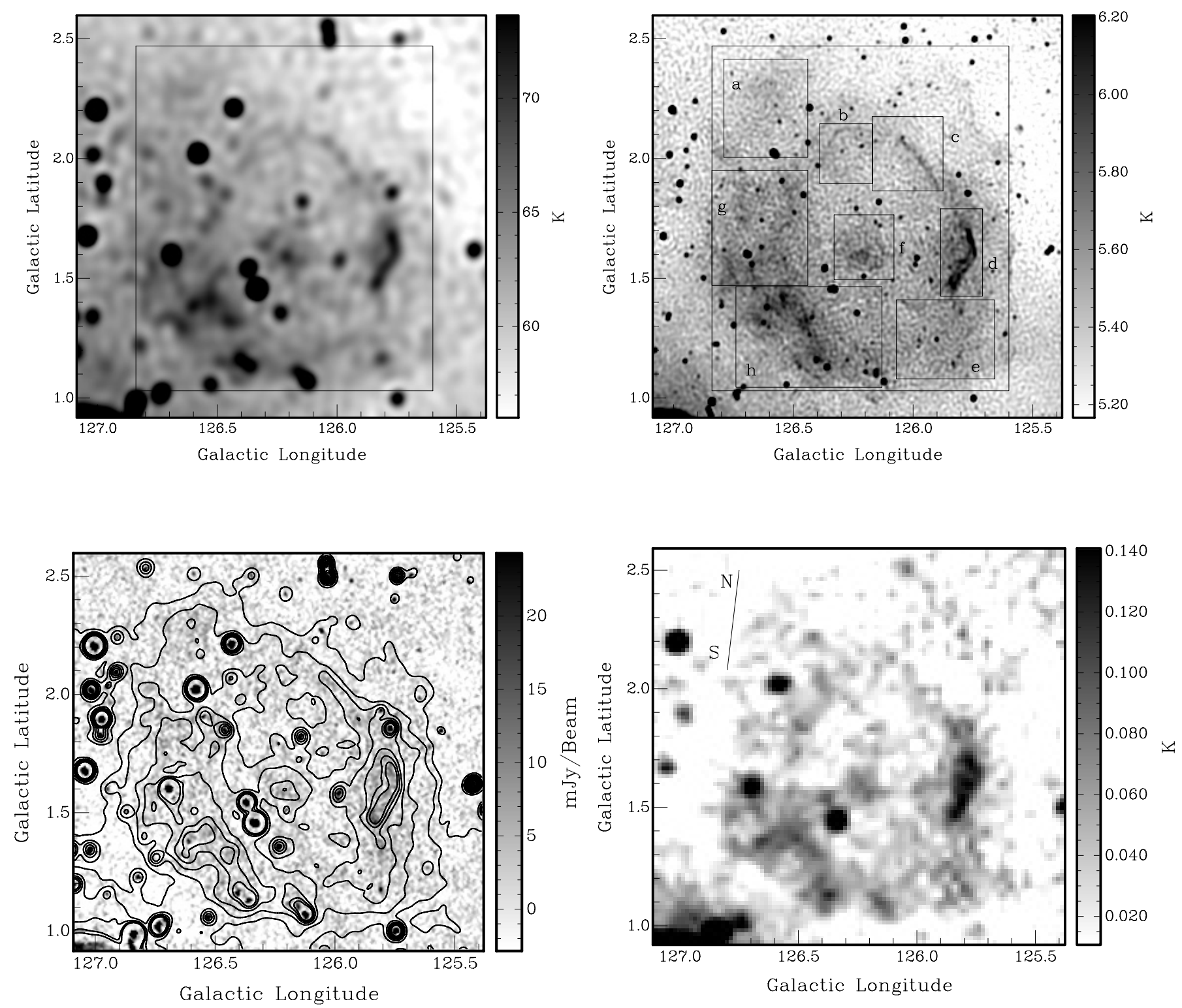

Fig. 1. The first row of images shows the CGPS maps at $408 \mathrm{MHz}$ (left) and $1420 \mathrm{MHz}$ (right). The left of the second row shows the WENSS image at $327 \mathrm{MHz}$ (grey scale) with contours from the $1420 \mathrm{MHz}$ map convolved to the same resolution as the $408 \mathrm{MHz}$ resolution (contours at 5.3, 5.4, 5.5, 5.6, 5.7, 5.8, 5.9 K). The right of the second row is the $2695 \mathrm{MHz}$ Effelsberg map. The box used for whole SNR T-T plots is shown in the upper left. The 8 boxes, labeled with letters and used for SNR sub-areas T-T plots, are shown in the upper right. The direction of North $(\mathrm{N})$ and South $(\mathrm{S})$ is marked on the lower right image.

\section{Results}

\subsection{Continuum emission from G126.2+1.6}

The CGPS continuum images at $408 \mathrm{MHz}$ and $1420 \mathrm{MHz}$ are shown in the upper left and right panels of Fig. 1. The lower left shows the WENSS (the Westerbork North Sky Survey, beam size $54^{\prime \prime} \times 54^{\prime \prime} \operatorname{cosec}(\delta)$, Rengelink et al. 1997) map at $327 \mathrm{MHz}$ (grey scale) with contours from the $1420 \mathrm{MHz}$ map convolved to the same resolution as the $408 \mathrm{MHz}$ map. The $2695 \mathrm{MHz}$ Effelsberg map is reproduced in the lower right for reference (Fürst et al. 1990). The Effelsberg map has an resolution of $4.3^{\prime}$ and a sensitivity of $50 \mathrm{mK} T_{B}$.
The $408 \mathrm{MHz}$ image for G126.2+1.6 reveals clearly more structure in comparison with Joncas's (1989) 408 MHz image, including a ring-like filamentary structure. Sharp and diffuse filamentary emissions are detected around the limb of G126.2+1.6, especially the bright western and northwestern sharp filaments and south to southeastern diffuse filamentary emissions. An area located at the center of the SNR shows significant diffuse emission. Compact sources distributed across the face of the SNR area are prominent, and illustrate the importance of having high enough spatial resolution to distinguish between SNR and compact source emission. The 1420 $\mathrm{MHz}$ map is the first published at this frequency and the best so far at any frequencies for G126.2+1.6. It shows much better detail of the fine structure in G126.2+1.6 than any previous 
Table 1. List of the brightest compact sources and their integrated Flux Densities (FD) inside G126.2+1.6.

\begin{tabular}{cccccccc}
\hline \hline Source num. & RA(2000) & Dec(2000) & GLONG & GLAT & FD at $408 \mathrm{MHz}$ & FD at $1420 \mathrm{MHz}$ & Sp. index \\
\hline 1 & {$[\mathrm{~h} \mathrm{~m} \mathrm{~s}]$} & {$\left[{ }^{\circ}{ }^{\prime \prime}\right]$} & $\mathrm{deg}$ & $\mathrm{deg}$ & $\mathrm{mJy}$ & $\mathrm{mJy}$ & $\alpha$ \\
\hline 2 & 12239.55 & 64724.2 & 126.331 & 1.458 & $412 \pm 15$ & $153 \pm 5$ & $0.80(0.76$ to 0.84$)$ \\
3 & 12535.93 & 643916.0 & 126.579 & 2.025 & $336 \pm 11$ & $132 \pm 4$ & 0.75 (0.72 to 0.79) \\
4 & 12426.25 & 645148.0 & 126.429 & 2.216 & $223 \pm 7$ & $63 \pm 2$ & 1.02 (0.98 to 1.05) \\
5 & 1265.20 & 641314.1 & 126.689 & 1.602 & $218 \pm 7$ & $91 \pm 3$ & 0.70 (0.66 to 0.74) \\
6 & 1235.06 & 641219.3 & 126.367 & 1.545 & $137 \pm 9$ & $54 \pm 3$ & 0.75 (0.69 to 0.82) \\
7 & 12248.99 & 634839.6 & 126.386 & 1.150 & $134 \pm 9$ & $82 \pm 11$ & 0.39 (0.28 to 0.52) \\
8 & 12019.86 & 634545.7 & 126.119 & 1.070 & $121 \pm 5$ & $38 \pm 2$ & 0.93 (0.88 to 0.98) \\
9 & 12358.46 & 634153.8 & 126.527 & 1.054 & $63 \pm 4$ & $22 \pm 1$ & 0.86 (0.80 to 0.94) \\
10 & 12138.46 & 6426.0 & 126.231 & 1.357 & $63 \pm 4$ & $16 \pm 1$ & 1.08 (1.00 to 1.16$)$ \\
11 & 12040.53 & 634747.8 & 126.153 & 1.108 & $58 \pm 5$ & $26 \pm 2$ & 0.65 (0.57 to 0.74) \\
12 & 11753.45 & 643447.7 & 125.767 & 1.854 & $54 \pm 5$ & $32 \pm 3$ & 0.43 (0.33 to 0.54) \\
13 & 1237.81 & 64173.8 & 125.985 & 1.580 & $49 \pm 5$ & $19 \pm 2$ & 0.75 (0.64 to 0.86) \\
14 & 12119.04 & 643029.9 & 126.141 & 1.823 & $46 \pm 4$ & $20 \pm 1$ & 0.66 (0.59 to 0.74) \\
15 & 12231.42 & 634737.6 & 126.356 & 1.129 & $37 \pm 8$ & $16 \pm 3$ & 0.65 (0.46 to 0.92) \\
16 & 12649.86 & 634940.5 & 126.823 & 1.224 & $37 \pm 3$ & $15 \pm 3$ & 0.74 (0.59 to 0.92) \\
17 & 12431.14 & 64254.6 & 126.542 & 1.409 & $33 \pm 5$ & $12 \pm 3$ & 0.79 (0.58 to 1.08) \\
18 & 12415.65 & 642948.3 & 126.457 & 1.850 & $28 \pm 6$ & $21 \pm 2$ & 0.22 (0.04 to 0.44) \\
19 & 12136.35 & 641633.9 & 126.199 & 1.596 & $31 \pm 4$ & $10 \pm 1$ & 0.91 (0.72 to 1.09) \\
\hline
\end{tabular}

images. A double filament structure appears in the northeastern part of the SNR, and multi-filamentary structures appear in the southeastern part. The inside edge of the filament distribution has a diamond-type shape more than a ring-type shape. The outlines of G126.2+1.6 at $408 \mathrm{MHz}$ and $1420 \mathrm{MHz}$ are very similar. The lower left of Fig. 1 shows that main features of G126.2+1.6 in the $327 \mathrm{MHz}$ WENSS map are consistent with the CGPS $408 \mathrm{MHz}$ and $1420 \mathrm{MHz}$ maps.

\subsection{T-T Plot spectral indices}

Bright compact sources affect the measured integrated flux densities for G126.2+1.6 and its measured spectral index. Thus we correct for the effects of compact sources. Table 1 lists properties of the 19 brightest compact sources which are detected within G126.2+1.6 at both $408 \mathrm{MHz}$ and $1420 \mathrm{MHz}$.

First we discuss spectral indices between $408 \mathrm{MHz}$ and $1420 \mathrm{MHz}$ based on the T-T plot method (Turtle et al. 1962). The principle of the T-T plot method is that spectral indices $\left(T_{v}=T_{\mathrm{o}} v^{-\beta}\right)$ are calculated from a fit of a linear relation to the $T_{1}-T_{2}$ values of all pixels within a given map region. $T_{1}$ is the brightness temperature of a map pixel at one frequency and $T_{2}$ is for the second frequency. The higher resolution image has been smoothed to the lower resolution for the T-T plot comparison. The brightness temperature spectral index $\beta$ is derived from the slope of the line. The error in spectral index is derived from the uncertainly in slope of the line. The flux density spectral index $\alpha\left(S_{v} \propto v^{-\alpha}\right)$ is related to $\beta$ by $\beta=\alpha+2$. Spectral index refers to flux density spectral index $\alpha$ in this paper unless specifically noted otherwise.

For the T-T plot analysis, first a single region for the whole SNR is used, as shown in Fig. 1. This region yields the T-T plots shown in Fig. 2. Three cases are considered: using all pixels including compact sources; using all pixels after subtracting Gaussian fits to the compact sources listed in Table 1 from the images; and excluding compact sources. The compact sources are bright compared to the SNR emission. Since the compact sources have a steeper spectrum than the SNR, they are seen in the T-T plot (Fig. 2 left panel) as the steeper lines of points extending to higher $T_{B}$. Subtracting compact sources from the image before making the T-T plot removes the lines of points associated with the compact sources (Fig. 2 middle panel). However artifacts remain in the T-T plot due to imperfect source subtraction, especially at $408 \mathrm{MHz}$. The next step is to completely remove regions of pixels including the compact sources from the analysis. Each region is taken to be a few beamwidths across, so that any contribution from the compact source is below $1 \%$ of the diffuse SNR emission. Thus any artifacts associated with the compact source are also removed. Generally, the last step produces the best results (rightmost plot of Fig. 2). Therefore, in further discussion, the second case will be not considered.

Next, smaller areas (labeled a to $\mathrm{h}$ in the upper right panel of Fig. 1) are selected to search for spatial variations in spectral index. Table 2 lists the results for two cases of analysis: including compact sources and removing compact sources. Visual inspection of the T-T plots confirms that the second method 

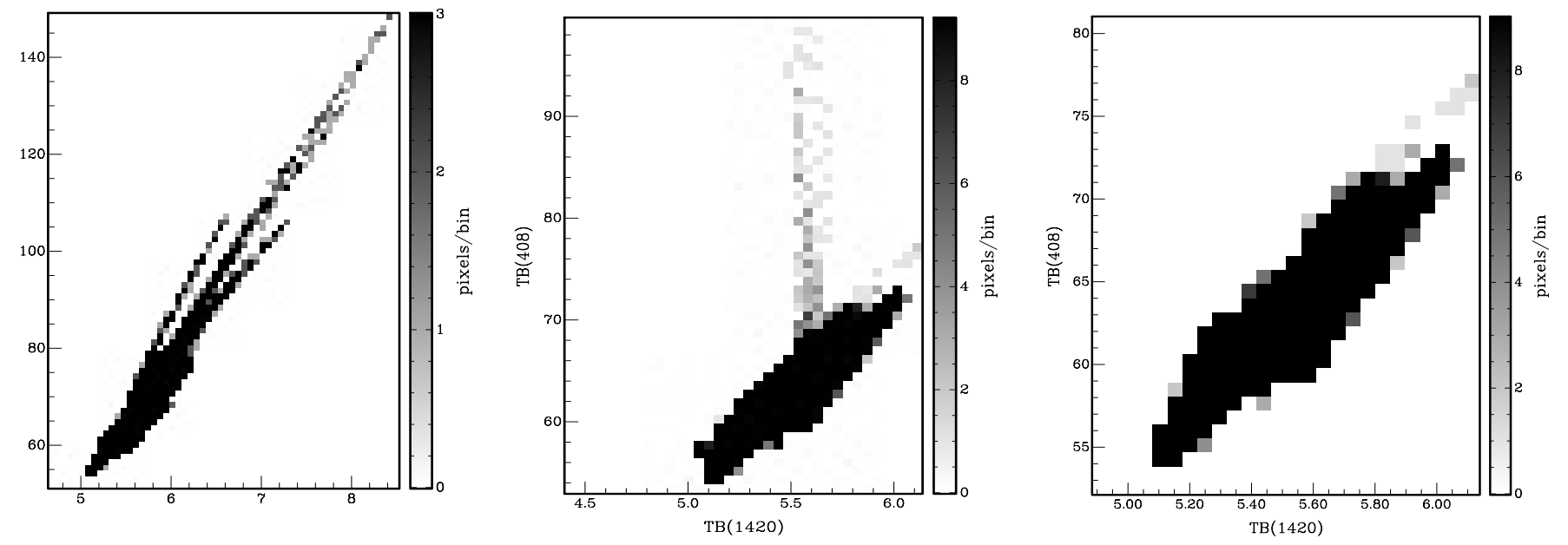

Fig. 2. Whole SNR 408-1420 MHz T-T plots. From left to right: plot for map including compact sources $(\alpha=0.42 \pm 0.09)$; plot for map with Gaussian fits to compact sources subtracted $(\alpha=0.31 \pm 0.11)$; plot for compact sources removed from analysis $(\alpha=0.30 \pm 0.08)$. Coordinates units of the left plots are same as the right plot.

Table 2. 408-1420 MHz T-T plot spectral indices with and without Compact Sources (CS).

\begin{tabular}{cccc}
\hline \hline Sp. index & $\alpha$ & $\alpha$ & $\alpha$ \\
\hline Area & Including CS & CS removed & Manual fit \\
\hline a & $0.39 \pm 0.33$ & $0.09 \pm 0.70$ & 0.20 \\
b & $0.36 \pm 0.23$ & $0.36 \pm 0.23$ & 0.46 \\
c & $0.46 \pm 0.09$ & $0.46 \pm 0.09$ & 0.45 \\
d & $0.59 \pm 0.02$ & $0.59 \pm 0.02$ & 0.59 \\
e & $0.45 \pm 0.55$ & $0.30 \pm 0.57$ & 0.41 \\
f & $0.64 \pm 0.10$ & $0.59 \pm 0.19$ & 0.57 \\
g & $0.34 \pm 0.14$ & $0.28 \pm 0.19$ & 0.29 \\
h & $0.50 \pm 0.14$ & $0.29 \pm 0.24$ & 0.19 \\
\hline All G126.2+1.6 & $0.42 \pm 0.09$ & $0.30 \pm 0.08$ & \\
\hline
\end{tabular}

Table 3. Integrated flux densities and spectral indices of G126.2+1.6 and compact sources within the SNR.

\begin{tabular}{cccc}
\hline \hline Freq. & G126.2+1.6 & CS of G126.2+1.6 & G126.2+1.6 and CS \\
$\mathrm{MHz}$ & $\mathrm{Jy}$ & $\mathrm{Jy}$ & $\mathrm{Jy}$ \\
\hline 408 & $9.7 \pm 3.9$ & $2.11 \pm 0.12$ & $11.8 \pm 4.0$ \\
1420 & $6.7 \pm 2.1$ & $0.84 \pm 0.06$ & $7.5 \pm 2.2$ \\
\hline$\alpha$ & $0.30 \pm 0.41$ & $0.74 \pm 0.07$ & $0.36 \pm 0.35$ \\
\hline
\end{tabular}

produces the most reliable results. The compact sources' influence on the spectral index calculation is obvious in the T$\mathrm{T}$ plots, and also seen in Table 2 for areas a, e, $\mathrm{f}, \mathrm{g}$ and $\mathrm{h}$. From now on we discuss spectral indices derived with compact sources removed, unless specified otherwise. From Table 2, we see spatial variation of spectral index within G126.2+1.6: $0.2-$ 0.6. Most difference are of low significance; the most significant difference is between $\mathrm{d}$ and $\mathrm{g}$ (at $1.6 \sigma)$.
Table 4. Integrated Flux Densities (FD) of G126.2+1.6 and of Compact Sources within G1262.+1.6 (CSFD).

\begin{tabular}{ccccc}
\hline \hline $\begin{array}{c}\text { Freq. } \\
\text { MHz }\end{array}$ & $\begin{array}{c}H P B W \\
\operatorname{arcmin}\end{array}$ & $\begin{array}{c}\text { FD } \\
\text { Jy }\end{array}$ & $\begin{array}{c}\text { CSFD } \\
\text { Jy }\end{array}$ & References for FD \\
\hline 83 & $59 \times 31$ & $35.0 \pm 7.0$ & $7.2 \pm 0.4$ & Kovalenko et al. (1994) \\
408 & $3.4 \times 3.8$ & $9.7 \pm 3.9^{*}$ & $2.1 \pm 0.1$ & this paper \\
408 & $3.5 \times 3.9$ & $12.0 \pm 2.5^{*}$ & & Joncas et al. (1989) \\
865 & $14.5 \times 14.5$ & $7.1 \pm 1.6$ & $1.2 \pm 0.1$ & Reich et al. (2003) \\
1420 & $1 \times 1.1$ & $6.7 \pm 2.1^{*}$ & $0.84 \pm 0.06$ & this paper \\
2695 & $4.4 \times 4.4$ & $4.4 \pm 0.4$ & & Fürst et al. (1984) \\
2695 & $4.3 \times 4.3$ & $3.7 \pm 0.4$ & $0.52 \pm 0.06$ & see text \\
4850 & $2.6 \times 2.6$ & $1.7 \pm 0.2^{* *} 0.34 \pm 0.07$ & Fürst et al. (1984) \\
\hline
\end{tabular}

* CS have been subtracted, ** lower limit.

\subsection{Integrated flux densities and spectral indices}

We have derived integrated flux densities of G126.2+1.6 from the $408 \mathrm{MHz}$ and $1420 \mathrm{MHz}$ maps. Values given have diffuse background subtracted. The resulting $408 \mathrm{MHz}$ to $1420 \mathrm{MHz}$ spectral index, using flux densities without compact sources, is $0.30 \pm 0.41$. Table 3 lists the flux densities and spectral indices of G126.2+1.6 and the compact sources within G126.2+1.6. Compact sources contribute about $18 \%$ at $408 \mathrm{MHz}$ and $13 \%$ at $1420 \mathrm{MHz}$ to the SNR's flux densities, and have a significant effect on the spectral index. It is noted that the whole SNR spectral index derived from integrated flux densities is consistent with the whole SNR spectral index $(0.30 \pm 0.08)$ derived by the T-T plot method.

Published integrated flux densities and errors for the SNR at other frequencies are given in Table 4. The errors are the published errors and don't include any uncertainty due to possible differences in flux density scales. We have calculated total compact source flux densities for these other frequencies, using the 408-1420 MHz spectral index upper and lower limits and flux densities from Table 1 . The compact sources' flux density 


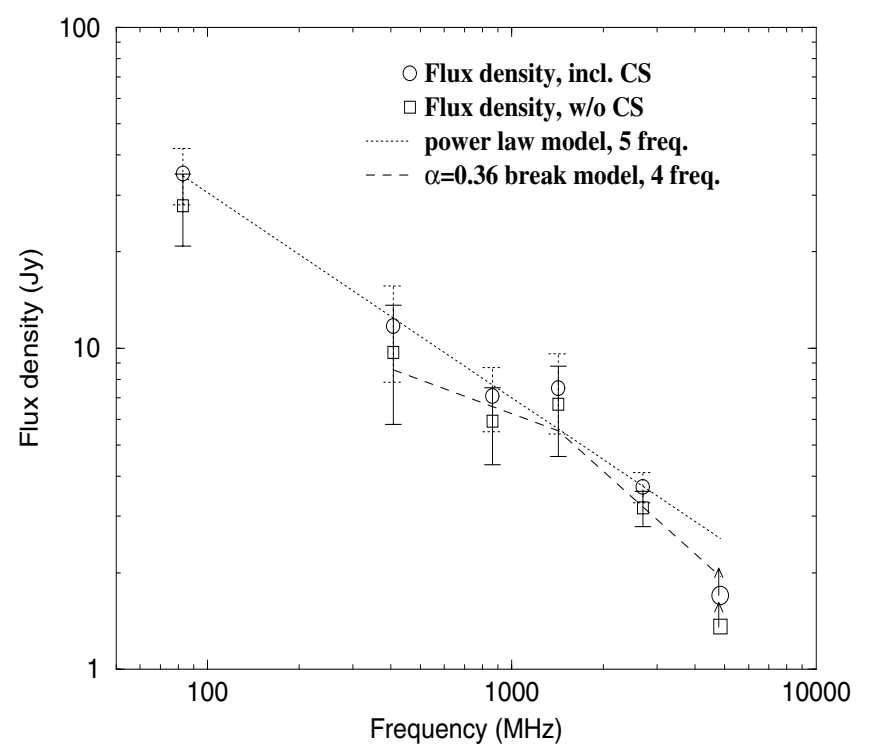

Fig. 3. Radio spectrum of G126.2+1.6. The value at $4850 \mathrm{MHz}$ is a lower limit. The power law model has a best-fit spectral index of 0.62 , $\chi^{2}=0.99$, for a fit to 5 frequencies $83-2695 \mathrm{MHz}$. The synchrotron aging model has a best-fit spectral index of 0.36 , break frequency of $1420 \mathrm{MHz}$ and $\chi^{2}=0.6$, for a fit to 4 frequencies $408-2695 \mathrm{MHz}$.

contribution is the highest at $83 \mathrm{MHz}$ at $21 \%$. It decreases to $18 \%$ at $408 \mathrm{MHz}, 17 \%$ at $865 \mathrm{MHz}, 13 \%$ at $1420 \mathrm{MHz}, 14 \%$ at $2695 \mathrm{MHz}$ and $20 \%$ at $4850 \mathrm{MHz}$. We have recalculated the flux density values of G126.2+1.6 at $83 \mathrm{MHz}, 865 \mathrm{MHz}$, $2695 \mathrm{MHz}$ and $4850 \mathrm{MHz}$ by subtracting the compact source flux density. For 408 and $1420 \mathrm{MHz}$ the values in Table 4 already have compact source flux density removed. We note that Fürst et al. (1984) gave a flux density based on the $2965 \mathrm{MHz}$ Effelsberg image with resolution $4.4 \times 4.4$ arcmin, but we obtain a new value from the Effelsberg $2695 \mathrm{MHz}$ image with a little higher resolution $4.3 \times 4.3 \mathrm{arcmin}: 3.7 \pm 0.4 \mathrm{Jy}$ and use this instead. We first fit the resulting flux density values with a power-law to obtain spectral index. Because the flux density at $4850 \mathrm{MHz}$ is a lower limit value, we do not include it in our fits. Figure 3 shows the corrected flux densities and the bestfit power-law. For the lowest 5 frequencies, there is a best fit spectral index of $\alpha=0.62$ with $\chi^{2}=0.99$. If we consider a synchrotron aging model for the G126.2+1.6 radio spectrum, the spectrum is steeper by 0.5 above the break frequency. The best fit to the lowest frequencies except $83 \mathrm{MHz}$ has $\alpha=0.36$, $\chi^{2}=0.6$ and a break frequency of $1420 \mathrm{MHz}$. A detail analysis about the spectrum fits has been given in discussion section.

\subsection{HI emission}

We have searched the CGPS radial velocity range for features in the HI which might relate to the morphology of G126.2+1.6. There is emission which is coincident with the boundary of G126.2+1.6 in the velocity range -32 to $-52 \mathrm{~km} \mathrm{~s}^{-1}$, and only in this velocity range. From -32 to $-42 \mathrm{~km} \mathrm{~s}^{-1}$, the HI emission is adjacent to the south and southeast edge of G126.2+1.6, and from -47 to $-52 \mathrm{~km} \mathrm{~s}^{-1}$, the $\mathrm{HI}$ emission is adjacent to the west edge. The spatial relation with the edge of G126.2+1.6 indicates that the HI is probably associated with the supernova remnant. These HI observations show a good association of HI features with G126.2+1.6, similar to other accepted HI associations with supernova remnants and with similar HI velocity ranges $\left(\sim 20 \mathrm{~km} \mathrm{~s}^{-1}\right)$, e.g. HI associated with the Cygnus Loop (Leahy 2003) and with DA530 (Landecker et al. 1999).

Figure 4 shows maps of HI emission in two channels (the left and middle panels are for radial velocities -32.8 and $-51.8 \mathrm{~km} \mathrm{~s}^{-1}$ respectively) and also integrated over channels from -32.8 to $-51.8 \mathrm{~km} \mathrm{~s}^{-1}$ (the right panel). Each map has superimposed on it contours of continuum emission at $1420 \mathrm{MHz}$ chosen to show G126.2+1.6.

\section{Discussion}

\subsection{The distance and age to $G 126.2+1.6$}

There are no previous reliable distance estimates for G126.2+1.6. Because of the low surface brightness of G126.2+1.6 and due to presence of compact sources, its flux density was measured with large errors. The surface brightness-diameter relations only gives a rough distance estimates (3.6 kpc to $7.1 \mathrm{kpc}$, Joncas et al. 1989). Joncas et al. (1989) discussed the distance in detail, suggested a range of 2 to $5 \mathrm{kpc}$, and noted that a reliable distance may be obtained based on a future measure of systemic radial velocity of the remnant, either from the optical lines or from detection of an interaction with the surrounding HI. The HI yields a radial velocity of $\mathrm{G} 126.2+1.6$ of $-42 \mathrm{~km} \mathrm{~s}^{-1}$. We take a galactic rotation curve with $R_{0}=8.5 \mathrm{kpc}, V_{0}=220 \mathrm{~km} \mathrm{~s}^{-1}$, and $V=250 \mathrm{~km} \mathrm{~s}^{-1}$ for $R>1.4 R_{0}$, and obtain a distance of $5.6 \mathrm{kpc}$ for $\mathrm{G} 126.2+1.6$. The range of distances obtained for radial velocities between $-39 \mathrm{~km} \mathrm{~s}^{-1}$ and $-45 \mathrm{~km} \mathrm{~s}^{-1}$ is 5.3 to $5.9 \mathrm{kpc}$.

At $5.6 \mathrm{kpc}$ distance, we get a mean radius of about $R=$ $64 \mathrm{pc}$ for G126.2+1.6. From the column density of the HI associated with G126.2+1.6, we estimate an approximate density $n_{0}=2 \times 10^{20} \mathrm{~cm}^{-2} /(2 R)=0.5 \mathrm{~cm}^{-3}$. Applying a Sedov model (e.g. Cox 1972), for a typical explosion energy of $E=$ $0.5 \times 10^{51} \mathrm{erg}\left(\epsilon_{0}=E /\left(0.75 \times 10^{51} \mathrm{erg}\right)=2 / 3\right)$, yields an age of $4.7 \times 10^{5} \mathrm{yr}$. This is too old since the SNR would have completely cooled by that time for $n_{0}=0.5 \mathrm{~cm}^{-3}$. The shock radius when the SNR has cooled is $R_{\mathrm{s}}^{(c)}=24.3\left(\epsilon_{0} / n_{0}\right)^{5 / 17} n_{0}^{-2 / 17} \mathrm{pc}$, and we require $R \leq R_{\mathrm{s}}^{(c)}$. Thus we find an upper limit on $n_{0}$ is $0.1 \mathrm{~cm}^{-3}$. This is consistent with the HI column density if the SNR exploded in a low density cavity and we are seeing the surrounding high density shell, as one sees for the Cygnus Loop (Leahy 2003). The revised Sedov age for G126.2+1.6 with $n_{0}<0.1 \mathrm{~cm}^{-3}$ is less than $2.1 \times 10^{5} \mathrm{yr}$. We also consider a post-Sedov model. In this case (Cox 1972), shocked gas cools and joins a dense shell which expands slowly, $\sim 30 \mathrm{~km} \mathrm{~s}^{-1}$, driven by the hot interior of the supernova remnant. However, for $n_{0}=0.5 \mathrm{~cm}^{-3}$ and the observed size of G126.2+1.6, the age of the supernova remnant is $\sim 10^{6} \mathrm{yr}$. This is too large, and requires that the pre-SNR density must be much lower. So again the conclusion is that the supernova exploded in a low density cavity in the interstellar medium, similar to the Cygnus Loop. 

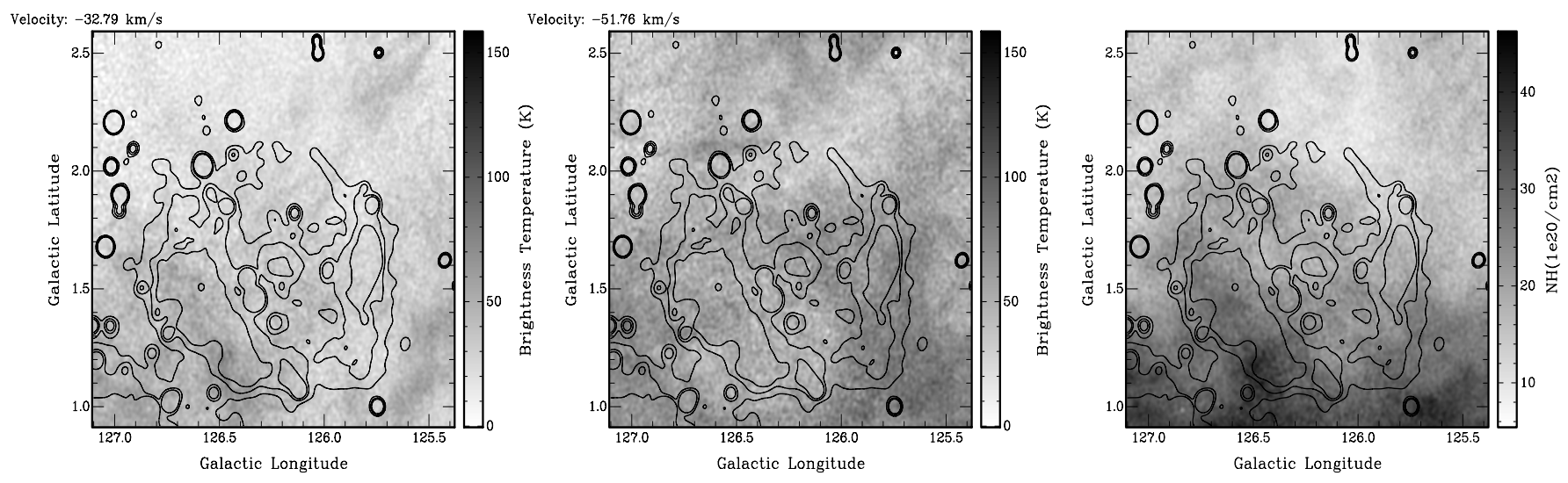

Fig. 4. Grey scale representation of HI emission in the field centered on G126.2+1.6. The radial velocity of the left and middle plots is indicated by the number at its top left corner. The grey scale bar of the left and middle plots has units of brightness temperature, of the right has units of

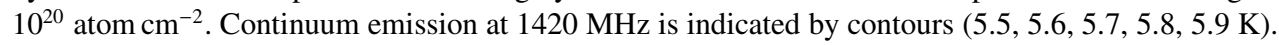

\subsection{Radio spectrum}

Generally, in the spectrum plots (Fig. 3), the $1420 \mathrm{MHz}$ flux density appears high and the $83 \mathrm{MHz}$ flux density appears high. Since our $1420 \mathrm{MHz}$ map is the best so far for G126.2+1.6 the $1420 \mathrm{MHz}$ one is the most reliable flux density. So we suspect that due to the faintness of the outer regions of G126.2+1.6, there is difficulty in determining the background level when the spatial resolution is poor. This results in increasing the uncertainty in flux densities.

The radio spectrum shown in Fig. 3 has a best-fit power-law with $\alpha=0.62$. This is much higher than the well-determined 408-1420 MHz T-T plot spectral index and the flux densitybased values (both about 0.30). Previous spectrum fits ( $\alpha=$ 0.48, Fürst et al. 1984; $\alpha=0.58$, Joncas et al. 1989; $\alpha=0.59$, Kovalenko et al. 1994; $\alpha=0.77$, Trushkin 2002) are not reliable due to inclusion of compact sources and poorer spatial resolution. For example we obtain $\alpha=0.36$ for $408-1420 \mathrm{MHz}$ spectral index if we include compact sources (see Table 3).

Here are some alternate explanations of the observed multifrequency radio spectrum. One alternative is that the $83 \mathrm{MHz}$ observation has too high a flux density. If we omit the flux density at $83 \mathrm{MHz}$, we obtain a power-law fit with $\alpha=0.61$ $\left(\chi^{2}=0.95\right)$. This still disagrees with the $408-1420 \mathrm{MHz}$ spectral index.

Another alternative is that the radio spectrum is described by a synchrotron aging model with resulting break in the radio spectrum. Fürst et al. (1984) gave a lower limit flux density at $4850 \mathrm{MHz}$ and argued the spectrum of G126.2+1.6 is steepening above $1 \mathrm{GHz}$. If we include all frequencies data (except the $4850 \mathrm{MHz}$ lower limit) we obtain $\alpha=0.59, \chi^{2}=0.95$ and break frequency $2240 \mathrm{MHz}$. If we use the synchrotron aging model and omit the flux density at $83 \mathrm{MHz}$, we obtain $\alpha=0.36$, $\chi^{2}=0.56$ and break frequency $1420 \mathrm{MHz}$. This is consistent with the $408-1420 \mathrm{MHz}$ index and is a better fit than the pure power-law model.

The last alternative we discuss, is that the curved spectrum is partly due to the spectral index variations within
G126.2+1.6. However, when we explicitly include the flux density from regions $\mathrm{c}$ and d, with their known $1420 \mathrm{MHz}$ flux density and known spectral index, we do not obtain significantly different results than above for either the power-law or synchrotron aging models.

In summary, we can reconcile the $0.30 \pm 0.08 \mathrm{~T}-\mathrm{T}$ plot spectral index with flux density-fit spectral index in two ways. (a) Including the $83 \mathrm{MHz}$ data, the radio spectrum is fit by a break spectrum with break frequency $1.6 \mathrm{MHz}$, and low frequency index of $\alpha=0.4$. This model is $1.3 \sigma$ worse than the synchrotron aging model with free $\alpha$; (b) omitting the $83 \mathrm{MHz}$ data, the radio spectrum is best fit by a model with break at frequency $1.4-1.5 \mathrm{GHz}$ with low frequency index $\alpha=0.36$.

\section{Conclusion}

We present high resolution and high sensitivity images of G126.2+1.6 at $1420 \mathrm{MHz}$ and $408 \mathrm{MHz}$ and show new HI-line emission data of G126.2+1.6 in this paper. The $1420 \mathrm{MHz}$ continuum image is the best image of G126.2+1.6 at any frequency yet. Flux densities at $408 \mathrm{MHz}$ and $1420 \mathrm{MHz}$ are obtained. The T-T plot spectral index is $0.30 \pm 0.08$ and the flux density-based spectral index is $0.30 \pm 0.41$ between $408 \mathrm{MHz}$ and $1420 \mathrm{MHz}$. By our analysis, a multi-frequency spectral index of about 0.36 is obtained using a synchrotron aging model. There is evidence at $1.6 \sigma$ for spatial variations in spectral index in G126.2+1.6 between about 0.3 and 0.6 . The association between HI brightness features and the SNR's structure suggests a distance of $5.6 \mathrm{kpc}$ for G126.2+1.6. The estimated Sedov age for G126.2+1.6 is less than $2.1 \times 10^{5} \mathrm{yr}$.

Acknowledgements. We acknowledge support from the Natural Sciences and Engineering Research Council of Canada. The DRAO is operated as a national facility by the National Research Council of Canada. The Canadian Galactic Plane Survey is a Canadian project with international partners. 


\section{References}

Cox, D. 1972, ApJ, 178, 159

Fürst, E., Reich, W., \& Steube, R. 1984, A\&A, 133, 11

Fürst, E., Reich, W., Reich, P., \& Reif, K. 1990, A\&AS, 85, 691

Haslam, C. G. T., Salter, C. J., Stoffel, H., \& Wilson, W. W. 1982, A\&AS, 47, 1

Higgs, L. A., \& Tapping, K. F. 2000, AJ, 120, 2471

Joncas, G., Roger, R. S., \& Dewdney, P. E. 1989, A\&A, 219, 303

Kovalenko, A. V., Pynzar, A. V., \& Udal'tsov, V. A. 1994, AR, 38, 95

Landecker, T. L., Routledge, D., Reynolds, S. P., et al. 1999, ApJ, 527, 866

Leahy, D. A. 2003, AJ, 586, 224
Leahy, D. A., \& Tian, W. W. 2005, A\&A, 440, 929

Reich, W., Kallas, E., \& Steube, R. 1979, A\&A, 78, 13

Reich, W., Reich, P., \& Fürst, E. 1990, A\&AS, 83, 539

Reich, W., Reich, P., \& Fürst, E. 1997, A\&AS, 126, 413

Reich, W., Zhang, X., \& Fürst, E. 2003, A\&A, 408, 961

Rengelink, R. B., Tang, Y., de Bruyn, A. G., et al. 1997, A\&AS, 124, 259

Taylor, A. R., Gibson, S. J., Peracaula, M., et al. 2003, AJ, 125, 3145 Tian, W. W., \& Leahy, D. A. 2005, A\&A, 436, 187

Trushkin, S. A. 2002, BSAO, 46, 62, http://cats.sao.ru

Turtle, A. J., Pugh, J. F., Kenderdine, S., \& Pauliny-Toth, I. I. K. 1962, MNRAS, 124, 297 